\title{
Estimation of tertiary dentin thickness on pulp capping treatment with digital image processing technology
}

\author{
Slamet Riyadi' ${ }^{1}$ Laila Ma'rifatul Azizah ${ }^{2}$, Fauri Hakim ${ }^{3}$, Cahya Damarjati $^{4}$, \\ Sartika Puspita ${ }^{5}$, Erma Sofiani ${ }^{6}$ \\ 1,2,3,4 Department of Information Technology, Faculty of Engineering, Universitas Muhammadiyah Yogyakarta, Indonesia \\ ${ }^{5,6}$ School of Dentistry, Faculty of Medical and Health Sciences, Universitas Muhammadiyah Yogyakarta, Indonesia
}

\section{Article Info}

Article history:

Received Oct 17, 2018

Revised Aug 17, 2019

Accepted Aug 30, 2019

\section{Keywords:}

Carries

Tertiary dentine

Pulp caping

B-spline

Image processing

\begin{abstract}
Dentists usually observe the tertiary dentin formation after pulp capping treatment by comparing periapical radiograph before and after treatment visually. However many dentists find difficulties to observe tertiary dentin and also they can't measure exactly the thickness of the tertiary dentin. The aims of this study is to assist the dentists to measure the area of tertiary dentin and calculate the dentin formation using b-spline image processing. The dental radiograph of 38 patients of pulp capping in the Dental Hospital Universitas Muhammadiyah Yogyakarta, Indonesia. Each of patient visited dental hospital 3 times. First, the patient got an application of pulp capping material. Second, after one-week treatment and temporary restoration and the third, after more than one month with the composite as the final restoration. Every visited the patient take a radiograph. Dentist placed the dot from the patient's radiograph. The dots were combined and processed with digital image processing. The b-spline method changed the dot to one area. After the calculation, the dentist can see whether there was dentin formation which means it is one of the treatment success indicators. Dentist has the better view to measure the dentin formation by providing area value of its tertiary dentin thickness calculation. We compare the result to the program calculation using the b-spline method and visual observation from the dentist. This study indicated the thickness of tertiary dentin can be measured by this program with an accuracy of $94.2 \%$. Therefore, dentist can make tertiary dentin thickness prediction from patient's radiograph.
\end{abstract}

Copyright @ 2020 Institute of Advanced Engineering and Science. All rights reserved.

\section{Corresponding Author:}

Slamet Riyadi,

Department of Information Technology,

Faculty of Engineering, Universitas Muhammadiyah Yogyakarta,

Brawijaya St., Tamantirto, Kasihan, Bantul, Yogyakarta, Indonesia.

Email: riyadi@umy.ac.id

\section{INTRODUCTION}

According to the Research on Fundamental Health Ministry of Health Republic of Indonesia in 2007 , the prevalence of caries is $72 \%$ of Indonesia's population where $45.5 \%$ of them are active caries that have not been treated. In 2013, dental caries in children 5-9 years was $28.9 \%$. This increase in 2007 which initially amounted to $21.6 \%[1,2]$. Various ways of cavities have been developed, ranging from the treatment, fillings, to extraction of the tooth. Pulp capping is the one of the endodontic treatment to maintain the pulp vitality by application of pulp capping material to stimulate tertiary dentin formation. Many pulp capping research has been done by many researchers to improve the pulp capping method [3-6]. Many of them use manual evaluation of pulp capping treatment by observing the periapical radiograph to observe the condition of filling, whether there are hermetic restoration and the thickness of tertiary dentin. 
Observing the tertiary dentin thickness, the dentist evaluate visually by comparing the results of radiograph before and after pulp capping treatment. They make a conclusion whether a formation tertiary dentin thickness after treatment. It shows one of the successful indication of pulp capping treatment. In addition to qualitative information, the quantitative data of dentin thickness is also indispensable as supporting data for next treatment. Some researcher already has done many types of research about pulp capping [7].

This fact becomes an opportunity for digital image processing technology to contribute and to assist the dentist in estimating the thickness of tertiary dentin to obtain the quantitative value. Computer-aided in dentistry has already developed in many cases. Jose already uses computation in morphometric mandibular index [8]. Some researchers proposed many tehniques to enhance radiograph images quality to ease diagnosis [9-12]. However, the teriary dentin thickness optimation hasn't been done.

Digital image processing technology has also been used in applications related to disease diagnosis and dental care. Caries detection using processing image has been performed by deformable template technique [13]. This technique is a polygon template that defines the general shape of human tooth shape. After each tooth was found its form and location, caries is identified with grayscale analysis method which is detecting the edges and segmenting among caries and tooth surfaces. The caries section will be recognized because of the radiographic image will be dark tinted while the tooth color is bright. In addition to detecting caries, other research also attempted to classify the types of caries, i.e., primary and secondary caries with a grayscale-based method of processing intensity [14].

Segmentation or separation between tooth objects and other parts get a lot of attention from researchers. The researchers propose the methods based on congruency of the local structure of the image [15]. This method was unaffected by changes in image size, rotation, translation, change of light and noise. Another method of texture-based extraction features using grey level co-occurrence matrix is also proposed to separate each tooth in the radiograph [16]. This method is used by many researcher, however it can not get good result for tertiary dentin thickness calculation. Supervised learning using Bayesian classifier was also developed [17].

Some of researchers using the b-spline algorithm to calculate the area in medical. The b-spline method widely uses in the medical field. Lehmann [18] used b-spline degree 2, 4, and 5 in image processing. Recently, Grove proves that b-spline is used in modelling of the medical image [19]. Some of them used in MRI and Echoradigraph. Segmentation and Tracking have been done by Pedrosa [20]. Pedrosa This various in $2 \mathrm{D}, 3 \mathrm{D}$, and $4 \mathrm{D}$ image. From the result above, b-spline is good enough to help make a curve for medical area which has many curves in the radiograph.

In dentistry, the use of b-spline has been done by Leung [21]. Leung minimizes the dental digital subtraction radiograph's bending mismatches with the b-spline method. Digital subtraction radiograph can improve disease detection. However, the bending and grid can lead mismatches to the radiograph. Therefore, b-spline can help to increase the accuracy of the radiograph's data. Digital image processing research related to dentistry is now widely developed. However, research studies on the use of image processing technology have not been used to assist the treatment of pulp caps yet, particularly to estimate the thickness of tertiary dentin. That is an opportunity to contribute to the application of image processing technology in the field of dentistry. On the other hand, this research would help dentists to overview the result of pulp capping efficiently and easily.

\section{RESEARCH METHOD}

Caries need to be restored for preventing the larger cavity in order the tooth can be maintained or no need to be extracted. Pulp capping treatment aims to protect the pulp from irritation and maintain vitality. Figure 1(a)-(c) are tooth illustrations with caries, after temporary restoration and after composite restoration, respectively. Observation of tertiary dentin thickness was done by comparing the thickness after treatment as shown in Figures 1(b) and Figure 1(c) with prior treatment as shown in Figure 1(a). The thickness of tertiary dentin after treatment is one of the indication of the successful of pulp capping treatment.

Ethical clearance standardized by The Medical Research Ethics Committee of Universitas Muhammadiyah Yogyakarta was implemented in this study. Subjects were the periapical radiograph of 38 pulp capping treatment patients. Each of patient visited dental hospital 3 times. First, the patient got an application of pulp capping material. Second, after one-week treatment and temporary restoration. Lastly, after more than one month with the composite as the final restoration. Informed consent signed by patients as a subject agreement. The research will be carried out in three main part: 1) collecting data, 2) the process of estimated tertiary dentin thickness and 3) result validation, as shown in Figure 2. Detailed 
explanations of each part of the study are as follows:

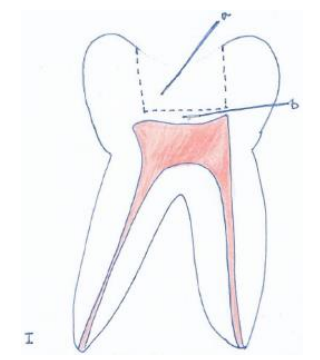

A. Caries (dx Pulpitis Reversible) B. Remaining Dentin Thickness

(a)

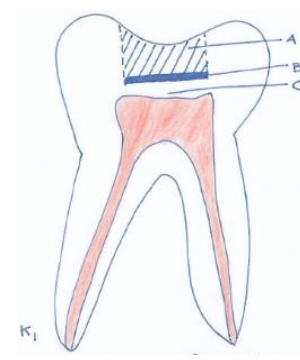

A. Temporary Filling B. Capping and Linning C. Tertiary Dentin

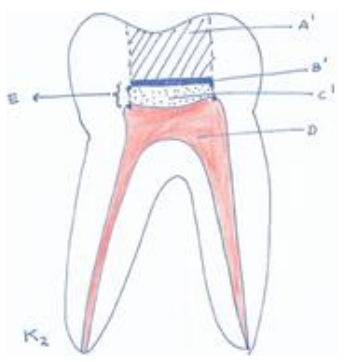

A'. Composite Filling

B'. Capping and Linning

C. Tertiary Dentin

D. Pulp Chamber

E. Tertiary Dentin Thickness

(c)

Figure 1. Illustration a tooth (a) with caries; (b) after treatment of temporary filling; and (c) after treatment of composite filling

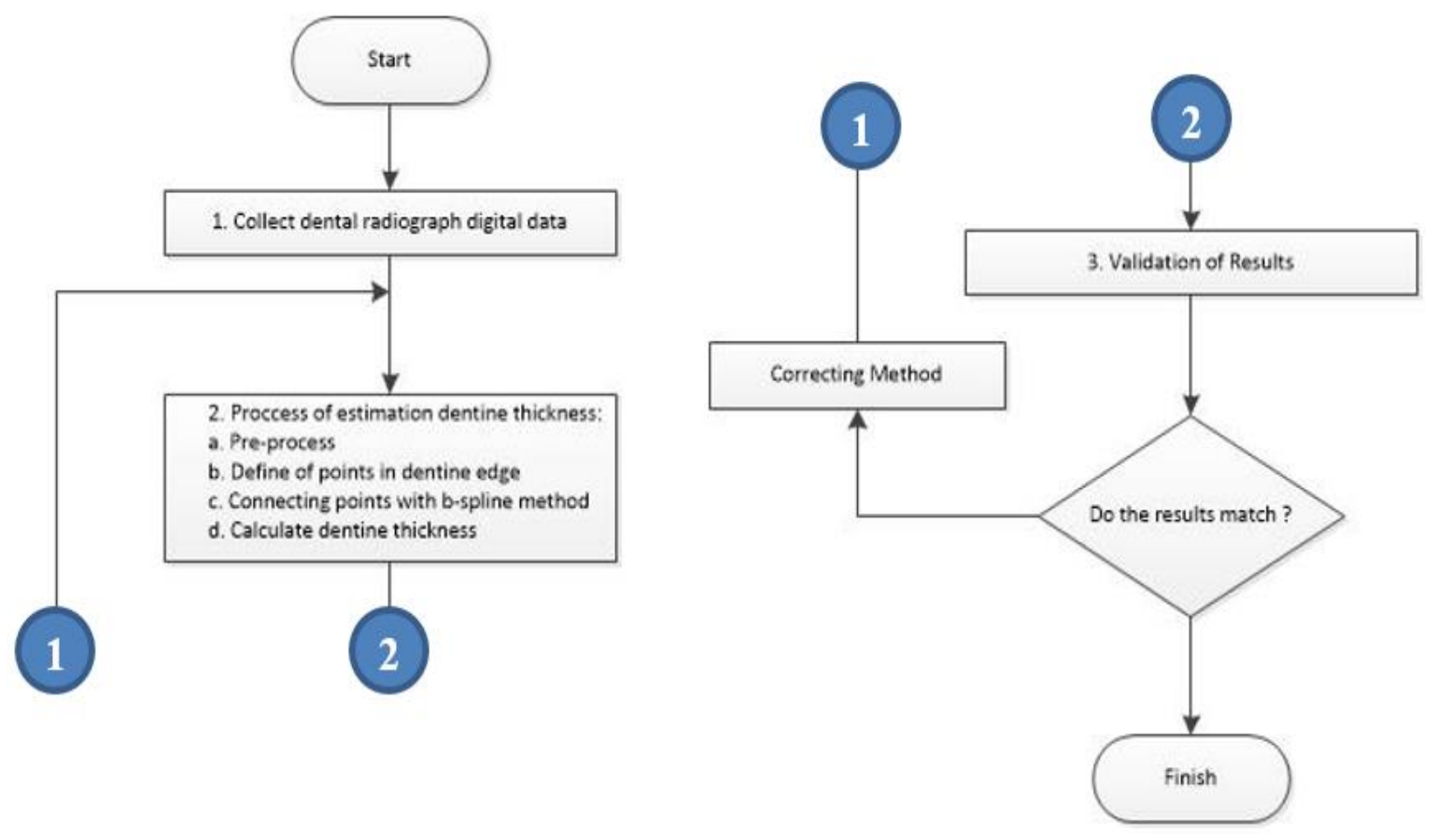

Figure 2. Method research

\subsection{Collecting data}

In this part, dental radiographic photos are obtained from the periapical radiograph data of patients whose do the pulp capping treatment at UMY Dental Hospital. Periapical radiograph should include photos before and after pulp treatment. Once it is collected, the data will be classified by dentist into three data sets in according to image quality, which is high, medium and low. Classification of data is done to facilitate the parts of making methods and analysis of results. The next part is the doctor determines the relevant points of tertiary dentin this is necessary because the points as a reference when doing the estimation process. The output of this part is the availability of dental radiograph digital image data, where the success indicator is the complete available data, covering all the photographs required when the pulp cage treatment evaluation is performed. 


\subsection{Process estimation tertiary dentin thickness}

The following explanation parts when making estimations:

\subsubsection{Pre-process}

The pre-processing part is preparing the image to be processed in a later part. This part consists of improving image quality through increased contrast and noise reduction, in particular aiming to clarify the edge of the tertiary dentin area. There are some photos of the same object but shot with a different distance; this will affect the calculation of the thickness estimation. Therefore, a solution is resizing on photographs that have different distances. When the photos have met the criteria, then the photos will be loaded into the program to perform the calculation process. The image is resized to 360x600 portrait image and 800x480 landscape image. The dentist also can adjust the position of the image that really fit their need.

\subsubsection{Define of point in dentin edges}

Fully automatic dentin area calculation is very complicated because of poor image quality, and the edge of the dentin area is not visible very clearly. In this study, the calculation is done semiautomatically which is dentist need to determine some starting points along the dentin route. The point determination is done by clicking on the desired dentin edge image as shown in Figure 3. Points that have been selected by the dentist will be symbolized as ' $x$ '. The dentist will determine the points as much as 6 - 8 dots. Point determination depends on the image to be estimated if the image has tertiary dentin with varying curvature then required the determination of the maximum point. The specified points are very influential in the calculation process.

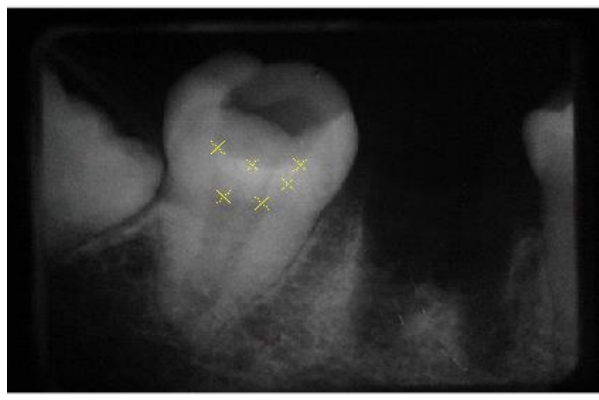

Figure 3. Image after choosing some point

\subsubsection{Connecting points with $b$-spline method}

The connection of the starting points is done automatically using the B-spline method. A B-spline is a generalization of the Bézier curve [22]. B-spline overcomes some of the drawbacks of Bézier curve, B-spline has a different approach to representing piecewise polynomial curves. To connect between points, we need a plot function. The plot function is used to connect point to point linearly. Plotting a function using the command, we calculated by the functions evaluation at $\mathrm{x}$-values $\mathrm{x} 1, \mathrm{x} 2, \ldots, \mathrm{xn}$ and making a curve that the points $\{(\mathrm{xk}, \mathrm{yk})\} \mathrm{nk}=1$, where $\mathrm{yk}=\mathrm{f}(\mathrm{xk})$ is passed through the curve [23]. The main $\mathrm{f}$ spacing in $\mathrm{B}$-form is the range that includes all clauses. This clauses mean that while the first node and the last nodes are perfect multiplicity by $\mathrm{k}$ and $\mathrm{k}$ is the spline's order. The $\mathrm{f}$ can be solved reliably at the end of the basic interval. The curve representation is generated by 'fnplt'. This representation is intended to first and last at the fixed point, despite everything of what is done otherwise, multiplexing is done when $f$ as spline. Yet, since they are zero B-splines except their support, B-form functions are zero exclude the basic range of that format. This is different from a function of a form whose value excluding the basic range of the form is given by the extensions to the left, more to the right, of each part of the polynomial [24].

The cubic spline curve is applied after the plot function. Eugene Lee is chosing the point results in spline curve with sequence point for the $j$-th point [25] centripetal scheme as the aggregated square root of chord length.

$$
\left.\sum_{i<j} \sqrt{\| p o i n t s}(:, i+1)-\operatorname{points}(:, i) \| 2\right)
$$

Periodic cubic spline curve is bulided if there isn't any repeated point. However, the corners result double points [26]. Using the cubic spline curve function will change the b-spline as shown in Figure 4. 
The cube spline forms an irregular shape that forms tertiary dentin as shown in Figure 5(a) and will be calculated the area.

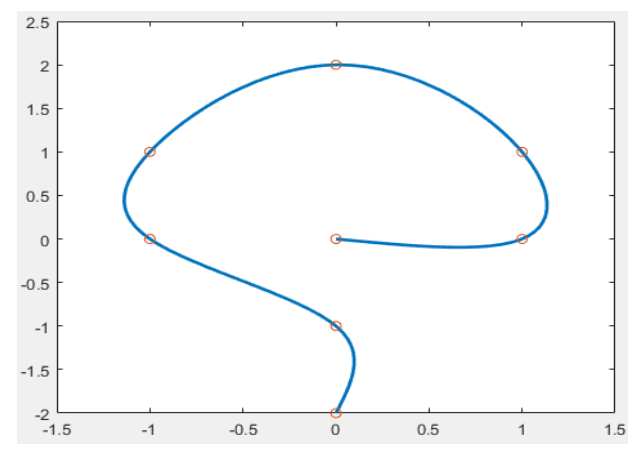

Figure 4. B-spline using cubic spline curve

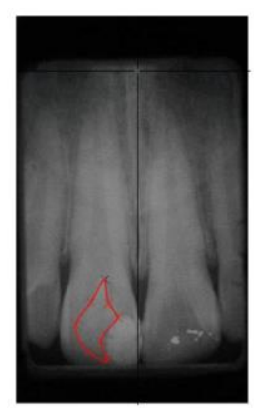

(a)

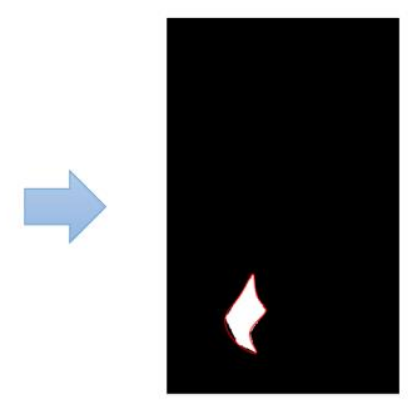

(b)

Figure 5. (a) Image with selected tertiary dentin area, (b) Convert to Binary Image

\subsubsection{Calculate dentin thickness}

Since the top and bottom edges of the tertiary dentin are not straight lines, the estimated tertiary dentin thickness is not calculated from the upper edge to the lower edge. The calculation is done by calculating the pixels' number in the tertiary dentin area. To be able to a calculation of pixel area is converted into the binary image, as in Figure $5 \mathrm{~b}$. A binary image ( 0 and 1 symbol image) is an array from a gray image. An image is composed to be discrete values that have been displayed in the array. The image area are marked with 1 , whereas the background is marked with 0 . Some of geometric and topological features can be calculated from the projected object region [27]. We can obtain the Binary image using poly2mask function for convert region of interest (ROI) polygon to region mask [28]. This function serves to separate the tertiary dentin area therefore that the tertiary dentin pixel value area is one whereas beyond it is worth 0 . Thus, the number of pixels is obtained by adding up all the pixels in the image.

\section{RESULTS AND ANALYSIS}

From the radiographic photographs of patients who examine the UMY dental hospital, the procedure that can be done by the dentist is calculated by the program. The treatment is a success when the result of the tertiary dentin after treatment is thicker than before previous untreated tertiary dentin. There are 38 patients with three photos each with varying length of time. The following sample radiographs are evaluated on the tertiary dentin area. From this 38 patients, our experiment use 114 picture to train as before and after treatment process. The validation process is used with comparing manual process by dentist.

Data that has been collected got refinement proses which the picture has wrong naming or cropping dental radiograph. The total picture is 114 pictures which 135 pictures are be tested to estimate the thickness of tertiary dentin.this section, it is explained the results of research and at the same time is given the comprehensive discussion. Results can be presented in Figures, graphs, tables and others that make the reader understand easily. The discussion can be made in several sub-chapters. From the patient's dental radiograph who got tooth repairment from different types of tooth or carries, the dentist is needed to identify the dot to dot of the tertiary dentin or pulp chamber. Figure 6 is the image of the before, in and after treatment.
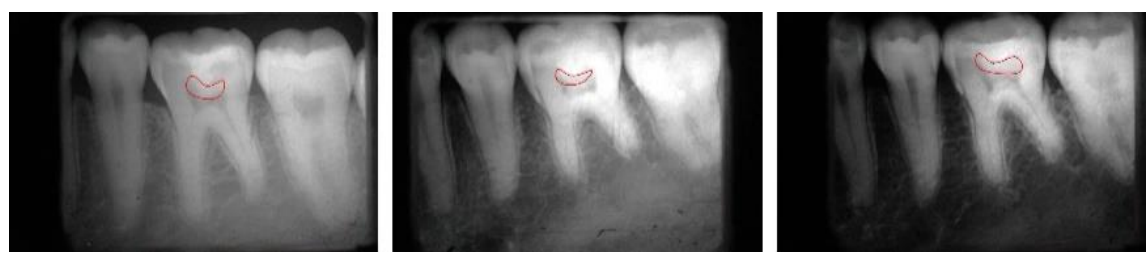

Figure 6. (left) Conditions of tooth prior to treatment of pulp caps, (middle) Conditions of tooth have been done capping pulp with temporary filling, (right) after treatment of temporary filling 
In Figure 6, we can see the dental image of the patient, it takes 8 points determined by the dentist to be able to get the maximum accuracy. In case, there are some photos that position when shooting is not fixed or the same. As a result, there is a change in the position of the tooth in the picture thus will affect the shape of the tertiary dentin area. Therefore, a solution that can be taken is to consult with dentist each photo to be estimated can maximize the accuracy when doing calculations.

The thickness calculation of tertiary dentin can be done by enumerating pixel value in the image. The image has been converted to the binary image. The value of binary image calculated ' 1 ' binary value in the area. Therefore, the area inside the line is tertiary dentin. From the radiograph and wire, the value will be:

$L 1$ pixel $=\left(\frac{\text { wide of wire }}{\text { wide of pixel }}\right)^{2}$.

$L$ tertiarydentine $=$ pixel value $x L 1$ pixel

From the equation (1) above, we can conclude the tertiary dentin value for this case is:

$L$ tertiarydentine $=$ pixel value $X\left(\frac{21.5}{434}\right)^{2}$

$L$ tertiarydentine $=$ pixel value $x 0.0025$

Therefore, we can get the tertiary dentin area as above value. Every Pixel area multiplies with 0.0025 to get the millimetre area. This equation to calculate the millimetre area of the dental radiography. From Figure 7, there are codes like "In", "K1" and "K2". The "in" code means that the patient has not been treated with the pulp cap. Code "K1" shows tooth that has been treated for pulp caps for several weeks, while the "K2" is the thickness code for several months after treatment. Tertiary dentin thickness increase depends on the duration of after treatment, but tertiary dentin thickness can grow only a few millimetres per month. This is the problem of the dentist when they want to know whether the treatment of pulp caps is satisfied or not with qualitative methods. Using quantitative method, the dentist will know faster, whether the dentin tertiary is increasing or not. The rising value can be concluded that the dentist succeeded in performing the treatment of the pulp cap to the patient. However, there is some data after the treatment of the thickness of the pulp thickness of tertiary dentin decreased, when it compares to dentin before treatment, this can occurs in because there is erosion can be elimination in caries or dead cells.

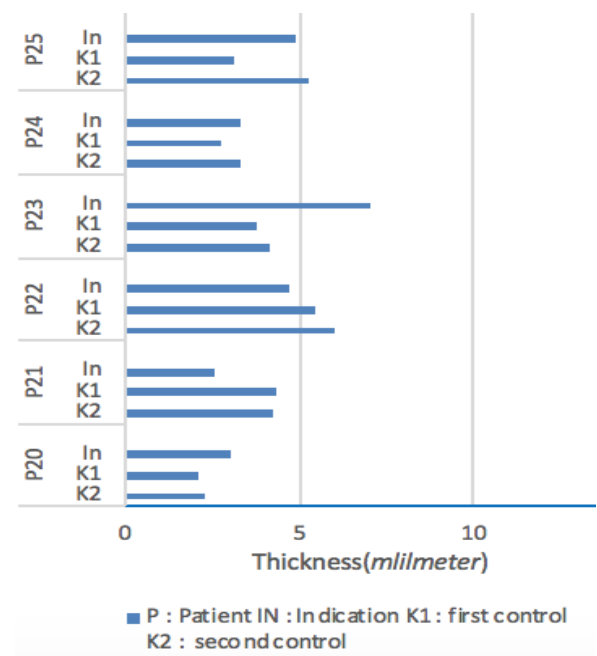

Figure 7.Graph tertiary dentin thickness from example patient P20-P25

From the result in Table 1, the manual observation from the dentist and the computerized inspection, there is almost no difference at all. There is only one patient's data that different with the computerized one from 15 patient's data and 45 periapical radiograph images. Although there is a patient that the dentin is decreasing, the result from manual and the digital one is same. However, there is a failed data that hardly interpreted by the system. This failure could happen because there is the different angle of dental radiography from "in" image to "k1" and " $\mathrm{k} 2$ " image. The different perspective of the picture can make the different judgment from the system to identify the thickness of the tertiary dentin. However, the dentist needs o place the dot in the right place, and that also does in every image of a radiograph. There would be distortion in 
the calculation. For example, P21, Dentist as expert's judgment there is thickness raising. However, the K2 radiograph isn't good enough that results in no improvement in the dentin thickness. Therefore, the data that failed because of two categories. First, there is no good quality of dental radiograph. Second, tertiary dentin thickness estimation is different with expert judgment.

Table 1. Result fo expert judgment on dental radiographs (example P20-P25)

\begin{tabular}{ccc}
\hline Nama & Code & \\
\hline & K2 & \\
& K1 & The dentin is increased with horm pulp resorption in distal tooth number 46. \\
In & K2 & Quality of K2 dental radiograph affected to tertiary dentin thickness. However, there is \\
& K1 & increasing thickness in tertiary dentin clinically because there is pulp area narrowing. The \\
& In & filling looks hermetic. \\
P22 & K1 & Goog fillings and hermetic. There is a narrower pulp chamber. \\
& In & \\
& K2 & \\
& K1 & Goog fillings and hermetic. There is a narrower pulp chamber. \\
& In & \\
P24 & K1 & The quality of K2 radiograph isn't good. The filling is good and hermatic. \\
& In & \\
& K2 & \\
P25 & K1 & Filling is just slightly hermatic \\
& In &
\end{tabular}

Table 2 is the result of the calculation which there is 3 bad or distortion of the dental radiograph that can lead to the different calculation. The bad quality of the dental radiograph or distortion will be emitted from accuracy calculation. However, this can help to improve the hospital quality dental radiograph assurance before it can be calculated in the program. From that table, we can conclude that there are 35 data with 2 failed data. This 2 data got the different result from expert/dentist analysis. From the table, the accuracy is $94.2 \%$.

Table 2. The result of calculation

\begin{tabular}{ccccccc}
\hline \multicolumn{2}{c}{ Number of patients } & & \multicolumn{4}{c}{ Computerized Result vs Dentist's } \\
Good images & Bad images & Total & Correct result & Wrong result & Total & Accuracy \\
\hline 35 & 3 & 38 & 33 & 2 & 35 & $33 / 35=94.2 \%$ \\
\hline
\end{tabular}

\section{CONCLUSION}

Our experimental result indicates that b-spline calculation through our program has the same value as the visual evaluation method by the dentist as expert's judge. The dentist will not be necessary to compare between photos, with the quantitative method the dentist can know how much tertiary dentin thickness increase than before. The quantitative method that can be obtained from image processing can define some points in the edge of tertiary dentin and will calculate in that area in millimeter. It has accuracy $94.2 \%$. In this research, the maximum marks that can be indicated in the edge are eight times and the minimum is six times.

Dental radiograph has low intensity and contrast which one that is the biggest problem in this research which can be done if the dental radiograph quality improving. Therefore, the calculation of the dentin tertiary thickness area is using a semi-automatic system. However, the weakness is the dentist must very careful when determining the point of the edge. Our future research will expand the developing software to calculate area with an automatic system, so the dentist is not necessary to define the point on the edge of tertiary dentin. For further, the research will develop a system in mobile phone apps that help the dentist to check their patient anytime and anywhere.

\section{ACKNOWLEDGEMENTS}

Authors would like to acknowledge Universitas Muhammadiyah Yogyakarta for the research grant (Multidisciplinary Research Scheme 2018) and Dental and Oral Hospital UMY for the provided radiograph data. 


\section{REFERENCES}

[1] Ministry of Health, "Research on Fundamental Health 2007", National Report 2007, 2007.

[2] Ministry of Health, "Research on Fundamental Health 2013", National Report 2013, 2013.

[3] S. Najeeb, et al., "Efficacy of Enamel Matrix Derivative in Vital Pulp Therapy: A Review of Literature," Iranian Endodontic Journal, vol. 12, no. 3, pp. 269-275, 2017.

[4] Nowicka, et al., "Clinical and Histological Evaluation of Direct Pulp Capping on Human Pulp Tissue Using a Dentin Adhesive System," BioMed Research International, vol. 2016, 2016.

[5] J.-B. Souron, et al., "Pulp Cell Tracking by Radionuclide Imaging for Dental Tissue Engineering," TISSUE ENGINEERING: Part C, vol. 20, no. 3, 2014

[6] S. Puspita, et al., "Nestin Expression of Exposed Pulp After Direct Pulp Capping by Calcium Hydroxide and Platelet Rich Plasma," Eur. J. Dent., vol. 10, no. 3, pp. 341-344, 2016.

[7] M. Lipski, et al., "Factors affecting the outcomes of direct pulp capping using Biodentine," Clinical Oral Investigations, 2017.

[8] J. Lopez-Lopez, et al., "Computer-aided system for morphometric mandibular index computation (Using dental panoramic radiographs)," Med Oral Patol Oral Cir Bucal, vol. 17, no. 4, pp. e624-e632, 2012.

[9] Jin-Woo Choi, Won-Jeong Han, Eun-Kyung Kim, 'Image enhancement of digital periapical radiographs according to diagnostic tasks', Imaging Science in Dentistry, 44, 31-35, 2014.

[10] Li G, Yoshiura K, Welander U, Shi XQ, McDavid WD, 'Detection of approximal caries in digital radiographs before and after correction for attenuation and visual response. An in vitro study', Dentomaxillofac Radiology 31, 113-16, 2002.

[11] Li G, Engström PE, Welander U., 'Measurement accuracy of marginal bone level in digital radiographs with and without color coding', Acta Odontol Scand 107, 254-58, 2007.

[12] Shi XQ and Li G, 'Detection accuracy of approximal caries by black-and-white and color-coded digital radiographs', Oral Med Oral Pathol Oral Radiol Endod 107 (433-436), 2009.

[13] Kantapanit, et. al., "Dental caries lesions detection using deformable templates," The 2001 IEEE International Symposium on Circuits and Systems, 2001.

[14] Oprea, Stefan, et al. "Image processing techniques used for dental x-ray image analysis," 31st International Spring Seminar on Electronics Technology 2008, 2008.

[15] Sattar, Farook and Karray, Fakhri, "Dental X-Ray Image Segmentation and Object Detection Based on Phase Congruene," 9th international conference on Image Analysis and Recognition, 2012.

[16] Rad, Abdolvahab Ehsani, et al., 'Digital Dental X-Ray Image Segmentation and Feature Extraction', TELKOMNIKA Indonesian Journal of Electrical Engineering, 11 (6), 3109-14, 2013.

[17] Lira, Pedro Henrique Marques, et al., 'Dental R-Ray Image Segmentation Using Texture Recognition', IEEE Latin America Transactions 12 (4), 694-98, 2014

[18] T. M. Lehmann, et al., "Addendum: B-spline interpolation in medical image processing," IEEE Transactions on Medical Imaging, vol. 20, no. 7, pp. 660-665, July 2001.

[19] A. Les Piegl, et al., "Heterogeneous modeling of medical image data using B-spline functions," Proceedings of the Institution of Mechanical Engineers, Part H: Journal of Engineering in Medicine, vol. 226, no. 10, pp. 737-751, October 2012.

[20] J. Pedrosa , et al., "Fast and Fully Automatic Left Ventricular Segmentation and Tracking in Echocardiography Using Shape-Based B-Spline Explicit Active Surfaces," IEEE Trans Med Imaging, vol. 36, no. 11, pp. 2287-2296, 2017.

[21] C. C. Leung, et al., "B-spline interpolation for bend intra-oral radiographs," Comput Biol Med., vol. 37, no. 11, pp. 1565-71, 2007.

[22] E. W. Weisstein, "B-Spline," MathWorld, [Online]. Available: http://mathworld.wolfram.com/B-Spline.html.

[23] P. Howard, MATLAB 7.8 Basics, 2009.

[24] T. MathWorks, "The MathWorks," [Online]. Available: https://www.mathworks.com/help/matlab/index.html. [Accessed 23 May 2017].

[25] E. T. Y. Lee, "Choosing nodes in parametric curve interpolation," Computer-Aided Design, no. 21, p. 363-370, 1989.

[26] T. MathWorks, "MathWorks," [Online]. Available: https://www.mathworks.com/help/curvefit/fnplt.html. [Accessed 22 May 2017].

[27] e. a. J. H. Sossa-Azuela, "Computing the Euler Number of a Binary Image Based on a Vertex Codification," vol. 11, pp. 360-370, 2013.

[28] T. MathWorks, "MathWorks," [Online]. Available: https://www.mathworks.com/help/curvefit/cscvn.html\#f7-5510. [Accessed 22 May 2017]. 


\section{BIOGRAPHIES OF AUTHORS}
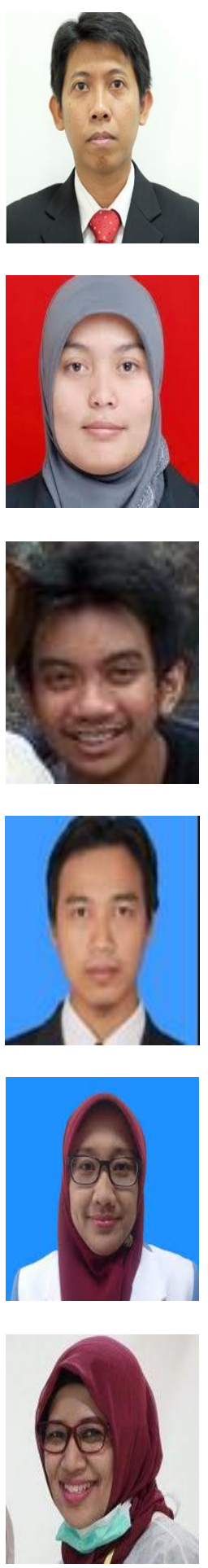

Slamet Riyadi obtained his Bachelor Degree in Electrical Engineering from the renowned Indonesian Gadjah Mada University, and both Master and $\mathrm{PhD}$ Degree from the National University of Malaysia in 2012. He joined Universitas Muhammadiyah Yogyakarta as a lecturer at the Department of Electical Engineering/Information Technology in 2001 and has published more than 40 research articles in international conferences and journals. $\mathrm{He}$ also served as reviewer for conferences and journals. His research interests are image processing and artificial intelligent.

Laila Ma'rifatul Azizah is a lecturer for the Department of Information Technology, Universitas Muhammadiyah Yogyakarta. She got her bachelor degree from Computer Science Universitas Gadjah Mada and master degree from Information Management National Taiwan University of Science and Technology. Her focus research is image processing, deep learing, ecommerce and ERP system for perishable food.

Fauri Hakim is Bachelor in Information Technology from Universitas Muhammadiyah Yogyakarta. He is professional worker in Icon+. His main research on image processing and programming language.

Cahya Damarjati obtained his Bachelor and Master Degree from Universitas Gadjah Mada. Currently he is a lecturer for the Department of Information Technology, Universitas Muhammadiyah Yogyakarta. His research interest is artificial intelligent and signal processing.

Sartika Puspita is lecturer for the Department of Dentistry, Universitas Muhammadiyah Yogyakarta. She obtained her Bachelor and Master Degree from Universitas Gadjah Mada. Her research interest is oral biology.

Erma Sofiani obtained her Bachelor in Dentistry and Professional Dentist from Universitas Gadjah Mada. She is currently lecturer for the Department of Dentistry, Universitas Muhammadiyah Yogyakarta. 